\title{
Roles of Sex Hormones and Gender in the Gut Microbiota
}

\author{
Kichul Yoon ${ }^{1}$ and Nayoung Kim ${ }^{2,3 *}$ \\ ${ }^{1}$ Department of Internal Medicine, Wonkwang University Sanbon Medical Center, Gunpo, Gyeonggi-do, Korea; ${ }^{2}$ Department of Internal \\ Medicine, Seoul National University Bundang Hospital, Seongnam, Gyeonggi-do, Korea; and ${ }^{3}$ Department of Internal Medicine and Liver \\ Research Institute, Seoul National University College of Medicine, Seoul, Korea
}

The distribution of gut microbiota varies according to age (childhood, puberty, pregnancy, menopause, and old age) and sex. Gut microbiota are known to contribute to gastrointestinal (GI) diseases such as irritable bowel syndrome, inflammatory bowel disease, and colon cancer; however, the exact etiology remains elusive. Recently, sex and gender differences in GI diseases and their relation to gut microbiota has been suggested. Furthermore, the metabolism of estrogen and androgen was reported to be related to the gut microbiome. As gut microbiome is involved in the excretion and circulation process of sex hormones, the concept of "microgenderome" indicating the role of sex hormone on the gut microbiota has been suggested. However, further research is needed for this concept to be universally accepted. In this review, we summarize sex- and gender-differences in gut microbiota and the interplay of microbiota and GI diseases, focusing on sex hormones. We also describe the metabolic role of the microbiota in this regard. Finally, current subjects, such as medication including probiotics, are briefly discussed.

(J Neurogastroenterol Motil 2021;27:314-325)

\section{Key Words}

Gastrointestinal diseases; Gender; Gut; Microbiota; Sex hormones

\section{Introduction}

Gut microbiota consists of as many as $10^{13}$ to $10^{14}$ microorganisms. ${ }^{1}$ The collective genome of microbiota (microbiome) covers various genes needed for many biologic processes; it is thus regarded as a separate organ for metabolism, like the human liver. ${ }^{2,3}$ Imbalance of the gastrointestinal microbiome could impact many diseases, such as neoplasm and autoimmune or cardiovascular conditions. ${ }^{4-6}$ There are several factors that affect the gut microbiome. Among them, gender and sex hormones play an important role after puberty. Interestingly, the bacteria-to-human cell ratio is dif- ferent between genders, that is, higher in women than in men. The bacteria-to-human cell ratio is 1.3 in men and 2.2 in women. ${ }^{8}$ As the gut microbiome is involved in the excretion and circulation process, the "microgenderome" indicating the interaction between sex hormones and the gut microbiome is an emerging research topic., 90 In functional gastrointestinal disorders (FGID), microbiota and inflammation can affect gastrointestinal (GI) function and sensation. ${ }^{11}$ There is emerging evidence that sex hormones can play a role in the interplay between the microbiome and GI symptoms. ${ }^{7}$ We hereby review the interaction between sex hormones, gender, and microbiota.

Received: September 14, 2020 Revised: February 16, 2021 Accepted: March 8, 2021

(a) This is an Open Access article distributed under the terms of the Creative Commons Attribution Non-Commercial License (http://creativecommons. org/licenses/by-nc/4.0) which permits unrestricted non-commercial use, distribution, and reproduction in any medium, provided the original work is properly cited.

*Correspondence: Nayoung Kim, MD, PhD

Department of Internal Medicine, Seoul National University Bundang Hospital, 82, Gumi-ro 173 beon-gil, Bundang-gu, Seongnam, Gyeonggi-do 13620, Korea

Tel: +82-31-787-7008, Fax: +82-31-787-4051, E-mail: nayoungkim49@empas.com 


\section{Estrogen and Microbiota}

Reproductive endocrine function involves a variety of hormones controlled by intricate feedback mechanisms (Fig. 1). ${ }^{12}$ The ovaries, adrenal glands, and adipose tissue produce estrogens. Estrogens produced in the body or ingested as food can be metabolized by gut microbes. The resultant metabolites again influence the host. ${ }^{13}$ Sex hormones directly modulate the metabolism of bacteria through steroid receptors, including estrogen receptor beta. ${ }^{14}$

Meanwhile, the gut microbiome with $\beta$-glucuronidase activity deconjugates the conjugated circulating estrogens excreted in the bile (Fig. 2). ${ }^{10}$ Deconjugation enables the estrogen reabsorption process to the system. ${ }^{10}$ The deconjugated estrogens circulate and affect multiple organs not only reproductive, but also skeletal, cardiovascular, and central nervous systems via estrogen receptors. ${ }^{15}$ Typically, estrogen binds to nuclear receptors, causing conformational change (Fig. 3). ${ }^{16}$ Central and peripheral alterations have been linked to irritable bowel syndrome (IBS) through intestinal barrier permeability and immune system modulation. ${ }^{9}$ Epidemiological studies suggest a female predominance of IBS, implying the

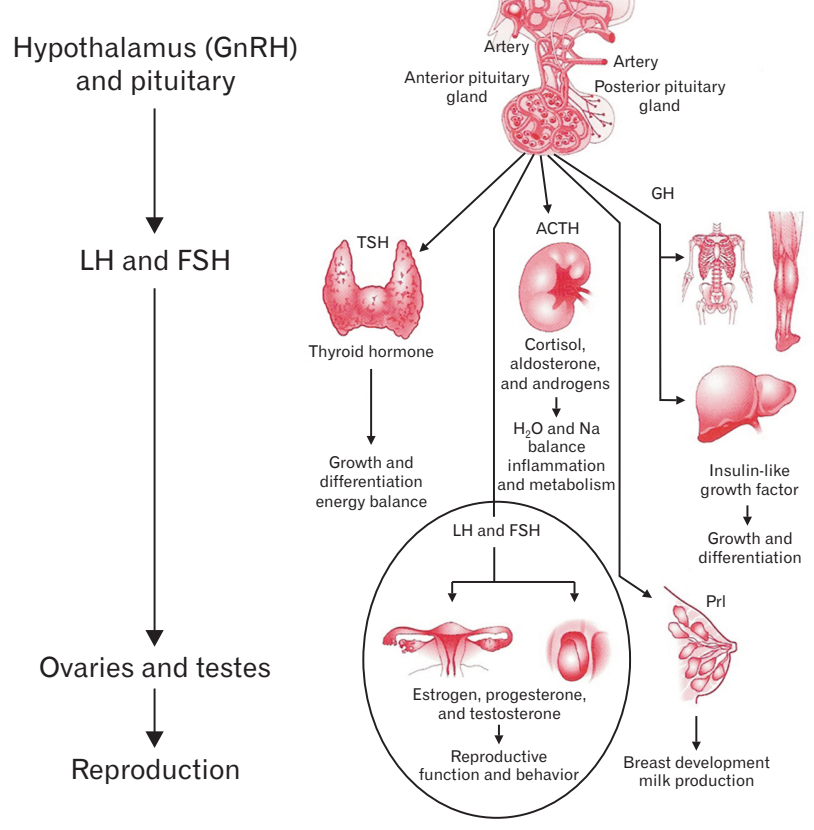

Figure 1. Overview of endocrine physiology. Gonadotropin-releasing hormone (GnRH). LH, luteinizing hormone; FSH, follicle-stimulating hormone; TSH, thyroid-stimulating hormone; ACTH, adrenocorticotropic hormone; GH, growth hormone; Prl, prolactin. Adapted from Molina. ${ }^{12}$ effect of sex hormones on its pathogenesis. ${ }^{9,17}$ GI transit duration also has been reported to vary according to menstrual cycle, pregnancy, and postpartum. ${ }^{17}$

In a human study, estradiol, estrone, 13 estrogen metabolites, and total estrogens were measured in urine and feces. ${ }^{18}$ In male and post-menopausal females, the total urinary estrogen levels were associated with fecal microbiota abundance and $\alpha$-diversity. ${ }^{18}$ Nonovarian estrogens were also associated with fecal Clostridia taxa and some Ruminococcaceae, which produce $\beta$-glucuronidase. Fecal $\beta$-glucuronidase was inversely correlated with fecal estrogens. ${ }^{18}$ In contrast, pre-menopausal female estrogen levels were not related to the fecal microbiome or enzymes. ${ }^{18}$ From the results they concluded that non-ovarian estrogens were affected by the intestinal microbiome and enzymes such as $\beta$-glucuronidase. ${ }^{18} \mathrm{~A}$ recent animal study showed that increasing age was associated with a significant reduction in fecal $\beta$-glucuronidase activity in females. ${ }^{19}$ Male $\mathrm{C} 57 \mathrm{BL} / 6$ mice showed significantly higher $\beta$-glucuronidase activity than did females. ${ }^{19}$ In this study, fecalase, a cell-free extract of feces, was employed in a colorimetric-based assay to quantify enzymatic activity. ${ }^{19}$ This approach could be employed to investigate gender differences in human samples.

Estrogen and testosterone have been shown to directly affect the gut microbiome and immune cells. $\beta$-estradiol affects the transformation of dendritic cells to produce IL-12 and IFN- $\gamma .^{20}$ This, in turn, activates pathways for pro-inflammatory cytokines. ${ }^{20}$ Estradiol prolongs the survival of $\mathrm{B}$ cells and activates polyclonal $\mathrm{B}$ cells. The resulting pro-inflammatory environment with altered intestinal gut permeability causes the migration of gut microbiota into the lamina propria, which again promotes inflammation processes. ${ }^{20}$

\section{Androgen and Microbiota}

The relationship between androgen and microbiota has been reported. An animal study measured unconjugated and glucuronidated androgen levels. In young adult males, unconjugated dihydrotestosterone (DHT) was 70-fold higher in the feces than in the serum. The distal intestine of germ-free mice showed high glucuronidated testosterone and DHT, but very low free DHT levels. They concluded that the gut microbiome affects the intestinal metabolism and deglucuronidation of androgens. ${ }^{21}$

Another study investigated the gut microbes and serum levels of testosterone in men and estradiol in women. ${ }^{22}$ Male and female patients with high testosterone or estradiol levels showed a more diverse gut microbiome. ${ }^{22}$

An animal study showed that antibiotic exposure inhibited 


\begin{tabular}{lcc}
\hline \multicolumn{1}{c}{ Genus } & $\beta$-glucuronidase & $\beta$-galactosidase \\
\hline Collinsella & + & - \\
Edwardsiella & + & - \\
Alistipes & + & + \\
Bacteroides & + & + \\
Bifidobacterium & + & + \\
Citrobacter & + & + \\
Clostridium & + & + \\
Dermabacter & + & + \\
Escherichia & + & + \\
Faecalibacterium & + & + \\
Lactobacillus & + & + \\
Marvinbryantia & + & + \\
Propionibacterium & + & + \\
Roseburia & + & + \\
Tannerella & + & + \\
\hline
\end{tabular}

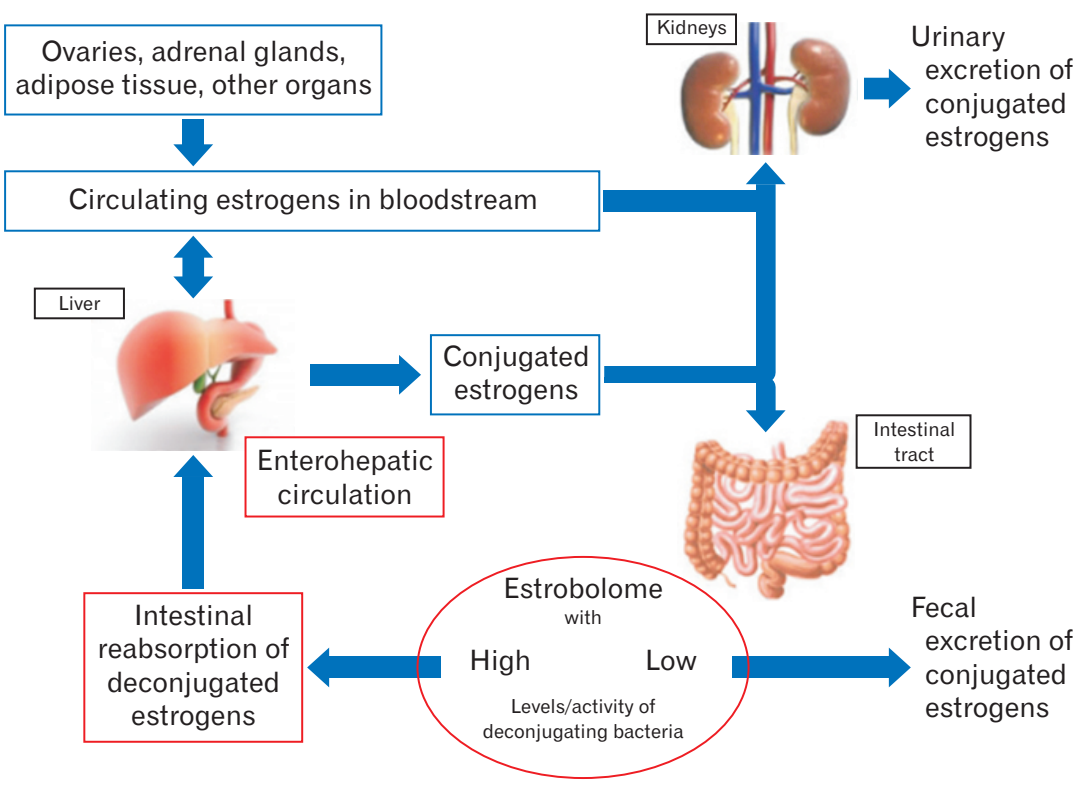

Figure 2. Estrobolome: gene repertoire of intestinal microbiota with the ability of estrogen metabolism. $\beta$-glucuronidase from microbiota deconjugates estrogen. The "active" deconjugated and unbound estrogens act on estrogen receptor alpha (ER $\alpha)$ and ER $\beta$. Adapted from Kwa et al. ${ }^{10}$

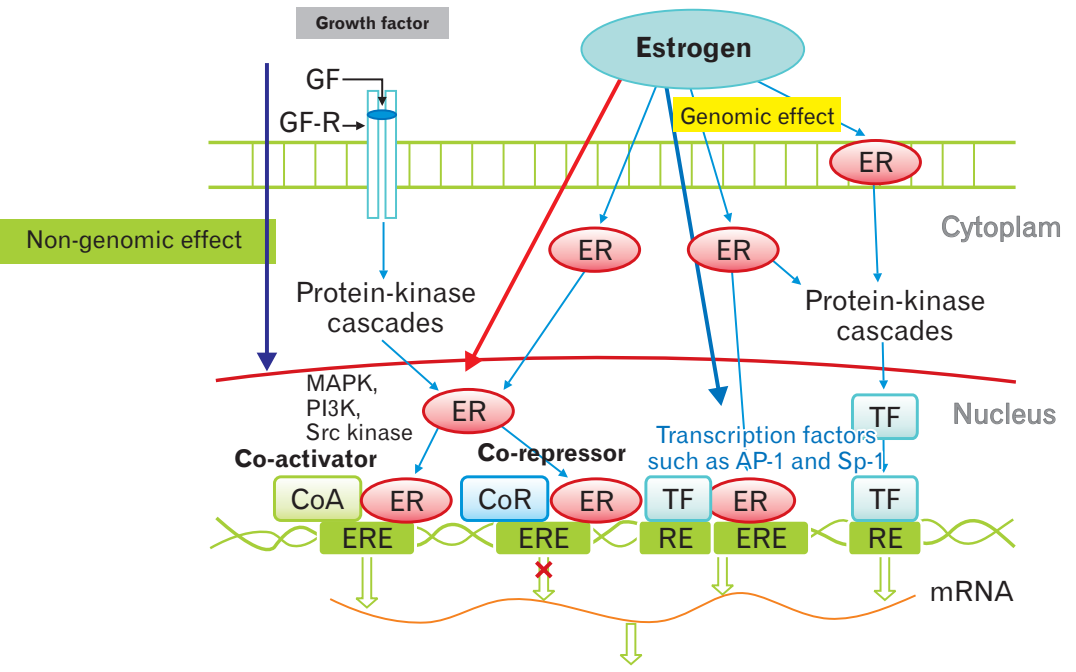

Protein products responsible for apoptosis, proliferation, adhesion, cell growth, signalling transdution, etc.

Figure 3. Molecular pathway of estrogen receptor (ER) action. Typically, estrogen binds to the ligand-binding domain of receptors in the nucleus, which causes a conformational change. Estrogen-responsive elements (ERE) mediate genomic effects. RE, response element; TF, transcription factor; GF, growth factor; GF-R, growth factor receptor; CoA, co-activator; CoR, co-repressor; MAPK, mictogen-activated protein kinase; PI3K, phophoinositide 3-kinase; Src, proto-oncogene tyrosine-protein kinase; AP-1, activating protein-1; Sp-1, stimulating protein-1. Adapted from Nie et al. ${ }^{16}$

mitochondrial dysfunction in a mouse Leydig tumor cell line (MLTC-1), inducing reduced steroidogenesis. ${ }^{23}$ Mice exposed to doxycycline subchronically showed a reduction in microbiome diversity and changes in composition. ${ }^{23}$
In males, testosterone shows an inhibitory effect on T-cell proliferation. In contrast to estradiol, testosterone does not alter the intestinal barrier. ${ }^{20}$ Blood testosterone levels are correlated with gut microbiota. Furthermore, the sex difference was reversed by male 


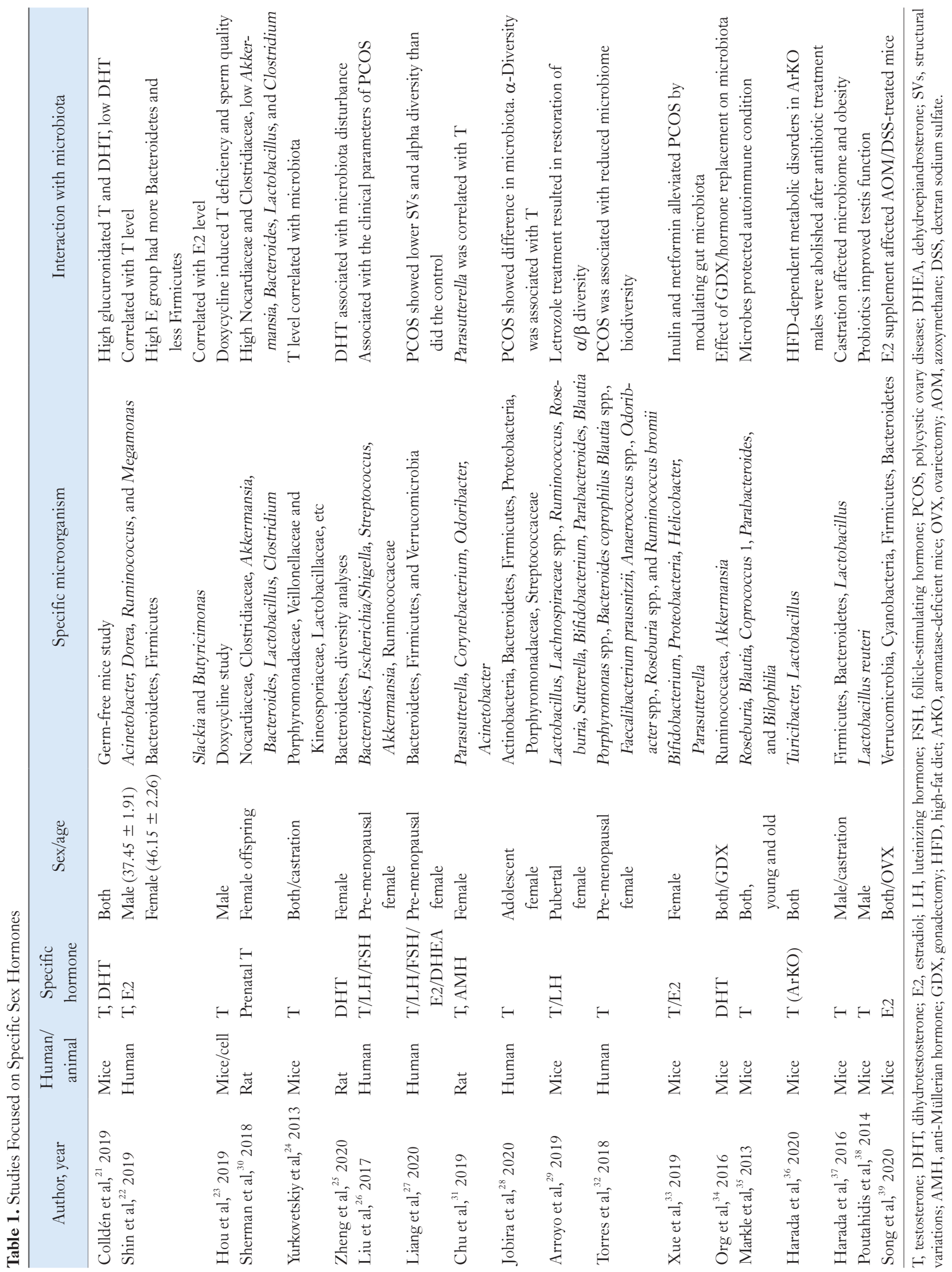


castration, which confirmed the effect of androgens on microbiota. ${ }^{24}$

Androgen excess represented by polycystic ovary syndrome (PCOS) is associated with gut microbiota disturbance. $5 \alpha$-Dihydrotestosterone (DHT) with a high-fat diet (HFD) could lower gut microbiota diversity. ${ }^{25}$ In a human study with 33 patients, the co-abundance bacterial groups increased in PCOS were Bacteroides, Escherichia/Shigella, and Streptococcus. ${ }^{26}$ They showed a negative correlation with ghrelin, while positive relationships with testosterone and body mass index were noted. In contrast, $A k$ kermansia and Ruminococcaceae decreased with PCOS, showed opposite results with body weight, sex hormone, and brain-gut peptides. $^{26}$

A recent human study showed that butyrate producers decreased in a PCOS group. ${ }^{27}$ Another human study with 58 obese female adolescents showed that gut microbiota was altered in obese adolescent PCOS patients. ${ }^{28}$ The aromatase inhibitor letrozole treatment of PCOS mice showed a positive effect on reproduction, metabolism, and the gut microbial community. ${ }^{29}$

In androgen metabolism, the gut microbiota has been reported to be an important regulator. In the small intestine content of mice, glucuronidated testosterone and DHT were found at high levels. The distal intestine had high levels of free $\mathrm{DHT}^{21}$ Markedly high levels of unconjugated DHT were observed in the feces of young adult males. ${ }^{21}$ In germ-free mice, glucuronidated testosterone and DHT were high; however, very low free DHT levels were noted in the distal intestine. This implies that gut microbiota affects the intestinal metabolism of DHT and testosterone.

We summarize the relevant studies focused on specific sex hormone in Table 1.

\section{Gender Difference/Gonadectomy Status -}

Important shifts in the maturation of the gut and sex hormones occur simultaneously. Sex differences within these systems occur at similar ages, implying communication between gender and gut microbiota. ${ }^{40}$ We summarize relevant studies focused on gender differences in Table 2.

Females showed greater gut microbial diversity in a study of 1135 individuals. ${ }^{41}$ In particular, Akkermansia muciniphila was abundant in females. ${ }^{41}$ In pre-menopausal women, compared to in post-menopausal female, the Firmicutes:Bacteroidetes ratio was higher, Lachnospira and Roseburia were relatively more abundant, and plasma levels of glucagon-like peptide-1, one of the intestinal incretins released from enteroendocrine cells in the gut to potentiate glucose clearance in response to the ingestion of food, were also higher. ${ }^{42}$ The relative abundances of the Prevotella, Parabacteroides, and Bilophila genera were lower in pre-menopausal than postmenopausal women. The plasma levels of IL-6 and monocyte chemoattractant protein-1 representing inflammatory levels were also lower than those in post-menopausal women. ${ }^{42}$

Gonadectomy (GDX) model was used in animal studies to investigate the function of sex hormones. A research showed that GDX showed that the abundance of Ruminococcacea was markedly different between GDX males and the control on a high-fat/ high-sucrose diet. ${ }^{34}$ In females, 2 strains showed differences between the sham control and GDX mice. Akkermansia was more abundant in the control than in GDX female mice. ${ }^{34}$ In addition, an interesting animal study with low capacity running (LCR) and high running capacity (HCR) rats was reported to mimic the postmenopausal phenotype. ${ }^{44}$ A decreased Firmicutes:Bacteroidetes ratio was observed in $\mathrm{LCR}$ - but not $\mathrm{HCR}$ - mice subjected to ovariectomy (OVX). LCR OVX was associated with increased microbial diversity and Bacteroidetes. HCR OVX did not show any changes in specific phyla. ${ }^{44}$

\section{Age}

Sex differences in childhood gut microbiota have not been reported owing to gonadal hormone quiescence. Approximate mean sex steroid levels in plasma shows pubertal surge and difference between sexes. ${ }^{40,65}$ The microbiota undergoes changes according to age, represented by a human study showing the core microbiota of elderly subjects was different from that of younger adults, with higher proportion of Bacteroides species (spp.) and distinct abundance patterns of Clostridium groups. ${ }^{66}$ A recent study reported that early-life adverse events such as maternal lipopolysaccharide injection, maternal separation, and unpredictable chronic stress affected gut microbiota according to gender. ${ }^{67}$

One germ-free mice study reported the sex-specific effects of the early-life microbiota status on serotonin production. ${ }^{44}$ The reduced sex differences in serotonin concentration in germ-free mice were reversed after microbial colonization. Sex differences in the hippocampal serotonergic neurocircuit also reappeared. ${ }^{40,68}$ Pubertal gonadal hormone alteration and its effect on sexually dimorphic brain development could not be reversed by hormone replacement in adulthood. ${ }^{69}$

The core microbiota of the elderly has a greater proportion of Bacteroides species and abundant Clostridium groups than does that of younger subjects. ${ }^{66}$ In centenarians, rearrangement in the Firmicutes population and an enrichment in facultative anaerobes 


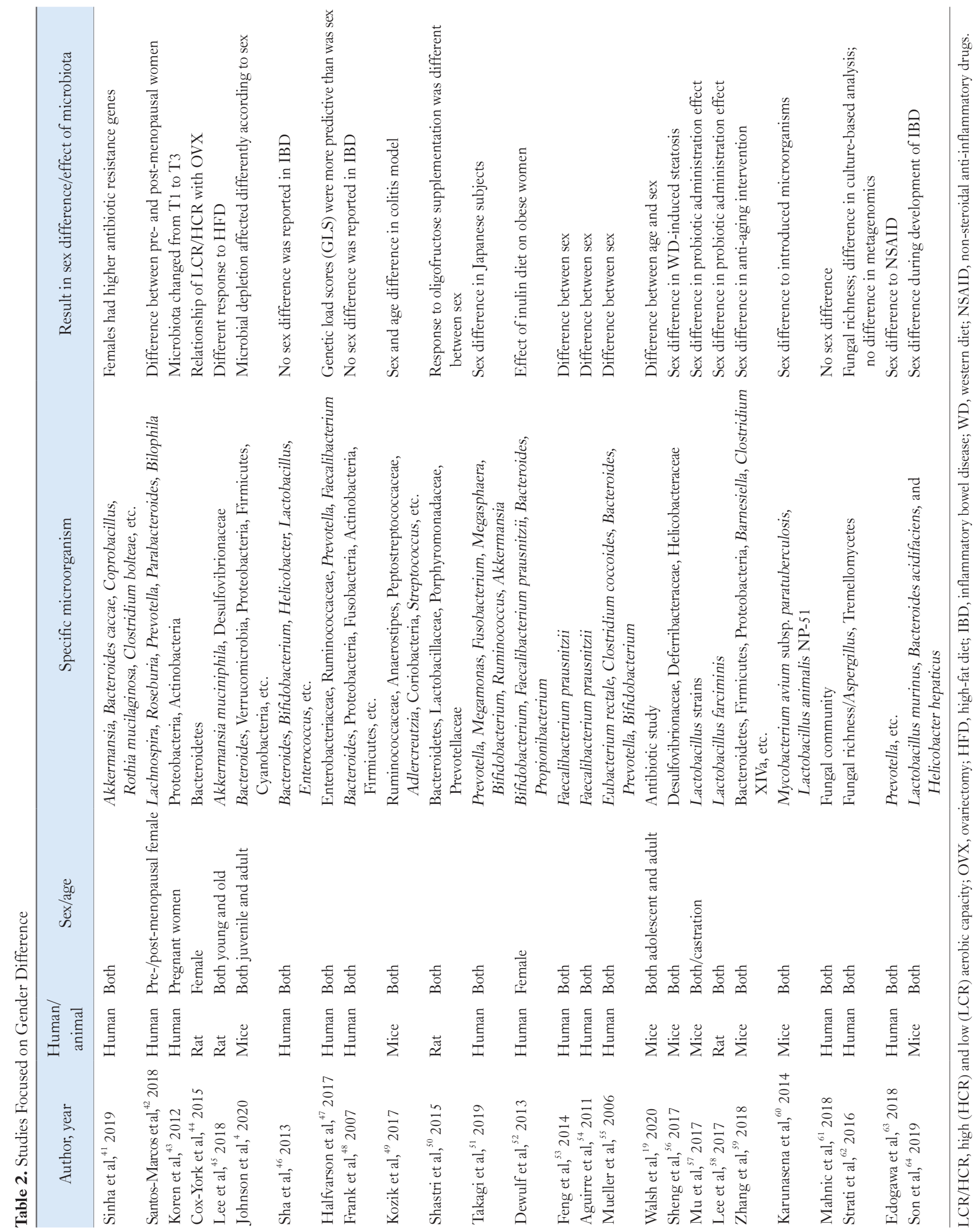


are characteristic. ${ }^{70}$ The old rats showed a higher abundance of Ruminococcaceae and Lachnospiraceae, which are butyrate producers. ${ }^{45}$ In the cecum of rats, the sulfate-producing Deltaproteobacteria increased with age. ${ }^{45}$ Recently, we reported the microbial changes and host response of F344 rat colon, focusing on sex and age. ${ }^{71}$ A HFD-induced low species richness and high abundance of Desulfovibrio spp. and Clostridium lavalense were noted with colon mucosal cell proliferation. ${ }^{71}$ Only in young rats and female aged rats, the abundance ratio of $A$. muciniphila and Desulfovibrio spp. increased with a HFD. ${ }^{71}$

In people aged over 70 years, changes in gut physiological function can affect the gut microbiome composition. ${ }^{7,72,73}$ In a German study with 35292 adults, total colony-forming units did not show age- or sex-related changes. ${ }^{52}$ However, individual bacterial species differed according to age: Escherichia coli and Enterococci spp. both increased, and Bacteroides spp. decreased. Lactobacillus and Bifidobacterium were stable throughout life. ${ }^{72,74}$

\section{Effect of Diet}

Diet has sex differential effects on gut microbiota. ${ }^{75}$ In the study of dietary intake of phytonutrients in relation to fruit and vegetable consumption in Korea, a higher proportion of women than men met the recommended intake of fruits and vegetables. ${ }^{76}$ In addition, the Korean national health and nutrition examination surveys among 3 groups, men, menopausal women, and postmenopausal women, showed that men's dietary carotenoid intake was the lowest. ${ }^{77}$ Since fruits and vegetables are the main source of dietary carotenoids, men consume less dietary fruits and vegetables than women. However, diet study is very difficult to perform in human especially in terms of sex differential effect of diet on the gut microbiota because sex- and gender-difference has many underlying conditions including age, race and culture. Usually diet studies are performed in animals, in which diet can be easily controlled. There are many studies on the relationship between gut microbiota and fat and/or bile acid regarding sex. HFDs induce an increase in bile secretion. ${ }^{78}$ Secondary bile acids are produced by gut bacteria from primary bile acids. Large intestinal anaerobic bacteria deconjugate and dehydroxylate to form secondary bile acids, such as deoxycholic acid and lithocholic acid. ${ }^{79,80}$ An animal study on sex differences in bile acids reported that sex differences in Western diet-induced steatosis, insulin sensitivity, and microbiota functions were farnesoid X receptor (FXR)dependent. ${ }^{56}$ Male Western diet-fed FXR knockout mice showed the most severe steatosis and highest liver and serum lipid profiles, and insulin resistance than females. FXR deficiency increased the numbers of Desulfovibrionaceae, Deferribacteraceae, and Helicobacteraceae only in males. ${ }^{56}$

In addition, in a study on laboratory fish, mice, and humans, Bolnick et $\mathrm{al}^{81}$ showed a gender-specific relationship between diet and microbiota. They first employed principal coordinate analysis and further analyzed the relative abundance according to diet and sex. They found that the mammalian gut microbiome is affected by sex-diet interactions. When males were fed a HFD, they showed an increased abundance of Lactobacillus, Alistipes, Lachnospiraceae, and Clostridium, whereas females did not. ${ }^{81}$

Another animal study investigated the association between gender, physical activity, and the gut microbiome. ${ }^{82}$ High-fat, high-corn starch, and high-sucrose diets were provided. Rats were housed in a sedentary environment or under physically active conditions with wheel-running access (RUN). Males in the RUN environment equally consumed the 3 diet types, with significantly less consuming the HFD than in the other groups. ${ }^{82}$ Females preferred a HFD. Regarding microbiota, only males showed an effect of physical activity. Males in the RUN showed more richness than did females in the sedentary environment. ${ }^{82}$

In IBS, the gut microbiota involved in bile acid metabolism may affect the pathophysiology.

A study on human fecal samples showed 2 orthologues, which has a role in secondary bile synthesis, were decreased in IBS patients. ${ }^{83}$ The microbiota, primary bile acids, and secondary bile acids (mainly deoxycholic acid and lithocholic acid) could be measured to investigate the sex differences in IBS in future studies.

\section{The Effect of Microbiota on the Disease Manifestations Depending on Sex Differences}

Gut dysbiosis triggers diseases that manifest differently between the sexes. The most investigated disease or condition include autoimmune diseases, obesity, and FGID. However, multifactorial factors including sex, age, and dysbiosis affect these diseases and they are also closely related and interact mutually. We tried to review relevant research to date.

\section{Functional Gastrointestinal Disorder and Microbiome-Gut-Brain Axis}

Gut microbiota play key roles in modulating the brain-gut axis and intestinal barrier (Fig. 4) ${ }^{8+86}$ Short-chain fatty acids (SCFAs) such as acetate, propionate, and butyrate are the most abundant 


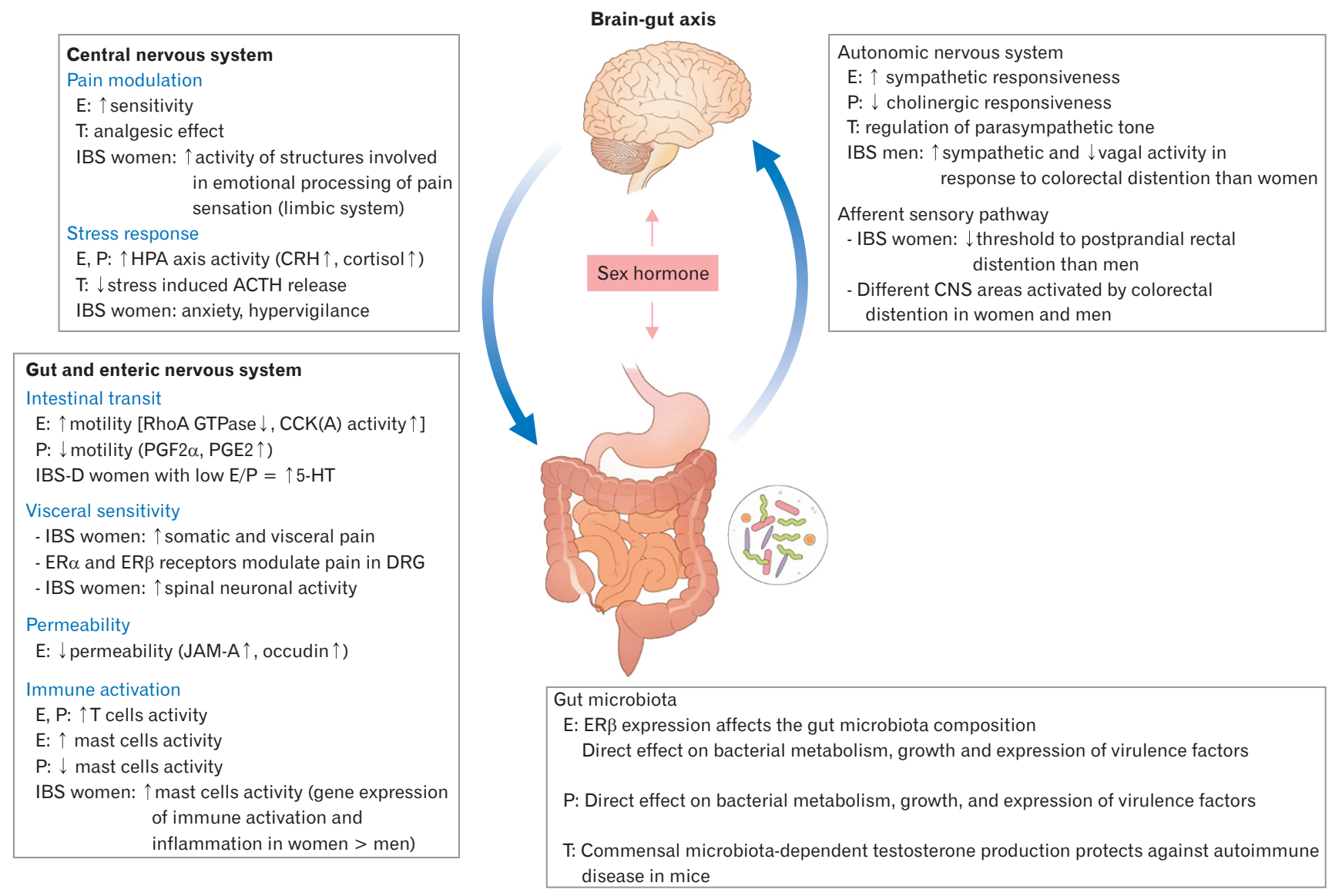

Figure 4. Sex hormone and brain-gut axis in irritable bowel syndrome (IBS). E, estradiol; T, testosterone; P, progesterone; HPA, hypothalamicpituitary-adrenal; CRH, corticotropin-releasing hormone; ACTH, adrenocorticotropic hormone; CNS, central nervous system; RhoA, Ras homolog family member A; GTP, guanosine-5'-triphosphate; CCK, cholecystokinin; PGE2, prostaglandin E2; IBS-D, IBS with diarrhea; 5-HT, 5-hydroxytryptamine; ER, estrogen receptor; DRG, dorsal root ganglion; JAM-A, junctional adhesion molecule-A. Adapted from Kim and Kim. ${ }^{84}$

and important microbial end-products. They are inflammation modulators that regulate gut motility and wound-healing. ${ }^{87}$ They have been also found as a link between the microbiome-gut-brain axis. ${ }^{6,80,88}$ Among them, butyrate is considered to be the reason for the health benefit from the fermentation process of indigestible carbohydrate. ${ }^{89}$ Interestingly, the sex difference in butyrateproducing gut microbiota has been reported. ${ }^{90}$ In addition, a recent study has shown that male and female rat gut microbiomes have different profiles of SCFAs when an oligofructose-containing diet is administered to both. ${ }^{50}$ In females, oligofructose supplementation increased the abundance of Bacteroidetes, while no difference was found in males. ${ }^{50}$ Furthermore, fecal butyrate, liver IgA, IL-6, and cecal IL-6 levels were increased in males but IL-10 levels were higher in females. ${ }^{50}$ Interestingly, Native Africans had remarkably higher abundance in the butyrate-producing bacteria such as Faecalibacterium prausnitzii, Clostridium cluster IV, and Clostridium cluster XIVa. ${ }^{91}$ In contrast, Bacteroides was the dominant in African Americans. ${ }^{91}$ Bacteroides-Prevotella group showed higher levels in men than women. ${ }^{55}$

There have been a few clinical studies regarding the microbiota and FGIDs. For instance, the differences in gut microbiota associated with sex were investigated in 277 Japanese volunteers. ${ }^{51}$ Increases in the genera Prevotella, Megamonas, Fusobacterium, and Megasphaera in males and increases in Bifidobacterium, Ruminococcus, and Akkermansia in females were reported. In females, 19.4\% had hard stools (Bristol stool form scale types 1 and 2 ), which was higher than that in male subjects. Loose-to-liquid stools (Bristol stool form scale type 6) were more common in male subjects. ${ }^{51}$ Tight junction proteins are important for the development of IBS and it contacts with microbiota and SCFA. Our team found that Helicobacter pylori was shown to be related to various tight junction proteins, particularly claudin-4 and occludin. ${ }^{92}$ Inter- 
estingly, an increase in claudin-2 was thought to be involved in male functional dyspepsia but not in females. ${ }^{92}$

\section{Medication and Probiotics}

There have been a few reports on medication and sex-difference of microbiota. A human study investigated 23 healthy men and women for differences in NSAID-induced intestinal barrier function and microbiome. ${ }^{63}$ The duodenal and fecal microbiota compositions were determined using $16 \mathrm{~S}$ ribosomal RNA sequencing. Healthy females had lower intestinal permeability, with higher duodenal and fecal microbial diversities than healthy males. Gut permeability increased after indomethacin administration in both sexes. ${ }^{63}$ However, only females showed decreased fecal microbial diversity, including an increase in Prevotella abundance, after indomethacin ingestion. The duodenal microbiota composition did not show sexspecific changes. ${ }^{63}$

Nowadays probiotics are popular for many reasons and it has been suggested that the effect could be different in terms of sex. Specifically, a probiotic clinical trial suggested an adjustment of the butyrate concentration by administering Lactobacillus paracasei DG. ${ }^{93}$ They showed that the effect of the probiotic on the microbiota and SFCAs correlated with the initial fecal butyrate concentration. $^{93}$ Another study reported that administration of a probiotic mixture of 5 Lactobacillus strains to lupus-prone mice improved renal function and showed anti-inflammatory effects in female and castrated male mice, but not in gonadally intact males, suggesting a sex difference in probiotic administration. ${ }^{57}$

In addition, female and male rats responded differently to probiotic treatment for repeated water avoidance stress-induced colonic microinflammation rat model. A 10-day treatment with Lactobacillus farciminis effectively treated female rats, but not males. ${ }^{58}$

Furthermore, supplementation of aged obese male mice with the probiotic Lactobacillus reuteri helped to restore testosterone levels, increasing seminiferous tubule cross-sectional profiles and spermatogenesis, and Leydig cell numbers, as well as decreasing IL-17 levels. The authors attributed these findings to the antiinflammatory properties of $L$. reuteri. ${ }^{38}$

Another study reported sex-based differences in the anti-aging effect of a tuna oil and algae oil mixture on the intestinal microbiota. ${ }^{59}$ Male mice showed better anti-aging effects than did female mice with a specific oil mixture ratio. ${ }^{59}$ The same oil treatment resulted in different intestinal microbiota composition alterations in different sexes. Lactobacillus and several butyrate producers were more abundant in males than in females. Some inflammation-relat- ed genera, such as Clostridium cluster XIVa, were lower in males. ${ }^{59}$

In addition, an animal study investigated the sex difference in the interaction between Mycobacterium avium subspecies (subsp.) paratuberculosis, probiotic Lactobacillus animalis NP-51, and the intestines. ${ }^{60}$ Host responses to $M$. avium subsp. paratuberculosis and $L$. animalis differed between sexes, especially the cytokines IL-1 $\alpha / \beta$, IL-17, IL-6, IL-10, and IL-12. Staphylococcus and Roseburia were consistently overrepresented in females compared to those in males. ${ }^{60}$

\section{Conclusions}

There has been significant progress in the research for relationship between gender, sex hormones, and gut microbiota. We tried to summarize recent studies on this subject under various conditions such as androgen excess, aging, GI inflammatory and functional diseases, nutrition, and medication. However, the majority of studies were performed in animals, so far. Further research on the interaction between gender, sex hormones, and gut microbiota may suggest novel preventive measures for relevant diseases.

Financial support: This research was supported by Support Program for Women in Science, Engineering and Technology through the Center for Women In Science, Engineering and Technology (WISET) funded by the Ministry of Science and ICT (No. WISET202003GI01). In addition, this work was supported by a grant from the National Research Foundation of Korea (NRF) funded by the government of the Republic of Korea (2019R1A2C2085149).

\section{Conflicts of interest: None.}

Author contributions: Kichul Yoon: design for contents and drafting the article; and Nayoung Kim: key concept and revision.

\section{References}

1. Gill SR, Pop M, Deboy RT, et al. Metagenomic analysis of the human distal gut microbiome. Science 2006;312:1355-1359.

2. Egert M, de Graaf AA, Smidt H, de Vos WM, Venema K. Beyond diversity: functional microbiomics of the human colon. Trends Microbiol 2006;14:86-91.

3. Possemiers S, Bolca S, Verstraete W, Heyerick A. The intestinal microbiome: a separate organ inside the body with the metabolic potential to influence the bioactivity of botanicals. Fitoterapia 2011;82:53-66.

4. Johnson BM, Gaudreau MC, Gudi R, Brown R, Gilkeson G, Vasu C. Gut microbiota differently contributes to intestinal immune phenotype 
and systemic autoimmune progression in female and male lupus-prone mice. J Autoimmun 2020;108:102420.

5. Cross TL, Kasahara K, Rey FE. Sexual dimorphism of cardiometabolic dysfunction: gut microbiome in the play? Mol Metab 2018;15:70-81.

6. Yoon K, Kim N. The effect of microbiota on colon carcinogenesis. J Cancer Prev 2018;23:117-125.

7. Kim YS, Unno T, Kim BY, Park MS. Sex differences in gut microbiota. World J Mens Health 2020;38:48-60.

8. Sender R, Fuchs S, Milo R. Revised estimates for the number of human and bacteria cells in the body. PLoS Biol 2016;14:e1002533.

9. Mulak A, Taché Y, Larauche M. Sex hormones in the modulation of irritable bowel syndrome. World J Gastroenterol 2014;20:2433-2448.

10. Kwa M, Plottel CS, Blaser MJ, Adams S. The intestinal microbiome and estrogen receptor-positive female breast cancer. J Natl Cancer Inst 2016;108:djw029.

11. Chey WD. The role of food in the functional gastrointestinal disorders: introduction to a manuscript series. Am J Gastroenterol 2013;108:694697.

12. Molina PE. Endocrine Physiology, 4th ed. New York, NY, USA: Lange medical Books/McGrow HIll 2013.

13. Chen KL, Madak-Erdogan Z. Estrogen and microbiota crosstalk: should we pay attention? Trends Endocrinol Metab 2016;27:752-755.

14. Menon R, Watson SE, Thomas LN, et al. Diet complexity and estrogen receptor $\beta$ status affect the composition of the murine intestinal microbiota. Appl Environ Microbiol 2013;79:5763-5773.

15. Paterni I, Bertini S, Granchi C, Macchia M, Minutolo F. Estrogen receptor ligands: a patent review update. Expert Opin Ther Pat 2013;23:1247-1271.

16. Nie X, Xie R, Tuo B. Effects of estrogen on the gastrointestinal tract. Dig Dis Sci 2018;63:583-596.

17. Meleine M, Matricon J. Gender-related differences in irritable bowel syndrome: potential mechanisms of sex hormones. World J Gastroenterol 2014;20:6725-6743.

18. Flores R, Shi J, Fuhrman B, et al. Fecal microbial determinants of fecal and systemic estrogens and estrogen metabolites: a cross-sectional study. J Transl Med 2012;10:253.

19. Walsh J, Olavarria-Ramirez L, Lach G, et al. Impact of host and environmental factors on $\beta$-glucuronidase enzymatic activity: implications for gastrointestinal serotonin. Am J Physiol Gastrointest Liver Physiol 2020;318:G816-G826.

20. Gomez A, Luckey D, Taneja V. The gut microbiome in autoimmunity: sex matters. Clin Immunol 2015;159:154-162.

21. Colldén H, Landin A, Wallenius V, et al. The gut microbiota is a major regulator of androgen metabolism in intestinal contents. Am J Physiol Endocrinol Metab 2019;317:E1182-E1192.

22. Shin JH, Park YH, Sim M, Kim SA, Joung H, Shin DM. Serum level of sex steroid hormone is associated with diversity and profiles of human gut microbiome. Res Microbiol 2019;170:192-201.

23. Hou X, Zhu L, Zhang X, et al. Testosterone disruptor effect and gut microbiome perturbation in mice: early life exposure to doxycycline. Chemosphere 2019;222:722-731.

24. Yurkovetskiy L, Burrows M, Khan AA, et al. Gender bias in autoimmu- nity is influenced by microbiota. Immunity 2013;39:400-412.

25. Zheng Y, Yu J, Liang C, Li S, Wen X, Li Y. Characterization on gut microbiome of PCOS rats and its further design by shifts in high-fat diet and dihydrotestosterone induction in PCOS rats. Bioprocess Biosyst Eng Published Online First: 10 March 2020. doi: 10.1007/s00449-02002320-w.

26. Liu R, Zhang C, Shi Y, et al. Dysbiosis of gut microbiota associated with clinical parameters in polycystic ovary syndrome. Front Microbiol 2017;8:324.

27. Liang Y, Ming Q, Liang J, Zhang Y, Zhang H, Shen T. Gut microbiota dysbiosis in polycystic ovary syndrome: association with obesity - a preliminary report. Can J Physiol Pharmacol 2020;98:803-809.

28. Jobira B, Frank DN, Pyle L, et al. Obese adolescents with PCOS have altered biodiversity and relative abundance in gastrointestinal microbiota. J Clin Endocrinol Metab 2020;105:e2134-e2144.

29. Arroyo P, Ho BS, Sau L, Kelley ST, Thackray VG. Letrozole treatment of pubertal female mice results in activational effects on reproduction, metabolism and the gut microbiome. PLoS One 2019;14:e0223274.

30. Sherman SB, Sarsour N, Salehi M, et al. Prenatal androgen exposure causes hypertension and gut microbiota dysbiosis. Gut Microbes 2018;9:400-421.

31. Chu W, Zhai J, Xu J, et al. Continuous light-induced PCOS-like changes in reproduction, metabolism, and gut microbiota in sprague-dawley rats. Front Microbiol 2019;10:3145.

32. Torres PJ, Siakowska M, Banaszewska B, et al. Gut microbial diversity in women with polycystic ovary syndrome correlates with hyperandrogenism. J Clin Endocrinol Metab 2018;103:1502-1511.

33. Xue J, Li X, Liu P, et al. Inulin and metformin ameliorate polycystic ovary syndrome via anti-inflammation and modulating gut microbiota in mice. Endocr J 2019;66:859-870.

34. Org E, Mehrabian M, Parks BW, et al. Sex differences and hormonal effects on gut microbiota composition in mice. Gut Microbes 2016;7:313322.

35. Markle JG, Frank DN, Mortin-Toth S, et al. Sex differences in the gut microbiome drive hormone-dependent regulation of autoimmunity. Science 2013;339:1084-1088.

36. Harada N, Hanada K, Minami Y, et al. Role of gut microbiota in sexand diet-dependent metabolic disorders that lead to early mortality of androgen receptor-deficient male mice. Am J Physiol Endocrinol Metab 2020;318:E525-E537.

37. Harada N, Hanaoka R, Hanada K, Izawa T, Inui H, Yamaji R. Hypogonadism alters cecal and fecal microbiota in male mice. Gut Microbes 2016;7:533-539.

38. Poutahidis T, Springer A, Levkovich T, et al. Probiotic microbes sustain youthful serum testosterone levels and testicular size in aging mice. PLoS One 2014;9:e84877.

39. Song CH, Kim N, Nam RH, Choi SI, Lee HN, Surh YJ. 17ß-estradiol supplementation changes gut microbiota diversity in intact and colorectal cancer-induced ICR male mice. Sci Rep 2020;10:12283.

40. Jašarević E, Morrison KE, Bale TL. Sex differences in the gut microbiome-brain axis across the lifespan. Philos Trans R Soc Lond B Biol Sci 2016;371:20150122. 
41. Sinha T, Vich Vila A, Garmaeva S, et al. Analysis of 1135 gut metagenomes identifies sex-specific resistome profiles. Gut Microbes 2019;10:358-366.

42. Santos-Marcos JA, Rangel-Zuñiga OA, Jimenez-Lucena R, et al. Influence of gender and menopausal status on gut microbiota. Maturitas 2018;116:43-53.

43. Koren O, Goodrich JK, Cullender TC, et al. Host remodeling of the gut microbiome and metabolic changes during pregnancy. Cell 2012;150:470-480.

44. Cox-York KA, Sheflin AM, Foster MT, et al. Ovariectomy results in differential shifts in gut microbiota in low versus high aerobic capacity rats. Physiol Rep 2015;3:e12488.

45. Lee SM, Kim N, Park JH, Nam RH, Yoon K, Lee DH. Comparative analysis of ileal and cecal microbiota in aged rats. J Cancer Prev 2018;23:70-76.

46. Sha $\mathrm{S}, \mathrm{Xu} \mathrm{B}$, Wang $\mathrm{X}$, et al. The biodiversity and composition of the dominant fecal microbiota in patients with inflammatory bowel disease. Diagn Microbiol Infect Dis 2013;75:245-251.

47. Halfvarson J, Brislawn CJ, Lamendella R, et al. Dynamics of the human gut microbiome in inflammatory bowel disease. Nat Microbiol 2017;2:17004.

48. Frank DN, St Amand AL, Feldman RA, Boedeker EC, Harpaz N, Pace NR. Molecular-phylogenetic characterization of microbial community imbalances in human inflammatory bowel diseases. Proc Natl Acad Sci USA 2007;104:13780-13785.

49. Kozik AJ, Nakatsu CH, Chun H, Jones-Hall YL. Age, sex, and TNF associated differences in the gut microbiota of mice and their impact on acute TNBS colitis. Exp Mol Pathol 2017;103:311-319.

50. Shastri P, McCarville J, Kalmokoff M, Brooks SP, Green-Johnson JM. Sex differences in gut fermentation and immune parameters in rats fed an oligofructose-supplemented diet. Biol Sex Differ 2015;6:13.

51. Takagi T, Naito Y, Inoue R, et al. Differences in gut microbiota associated with age, sex, and stool consistency in healthy Japanese subjects. J Gastroenterol 2019;54:53-63.

52. Dewulf EM, Cani PD, Claus SP, et al. Insight into the prebiotic concept: lessons from an exploratory, double blind intervention study with inulin-type fructans in obese women. Gut 2013;62:1112-1121.

53. Feng J, Tang H, Li M, et al. The abundance of fecal Faecalibacterium prausnitzii in relation to obesity and gender in Chinese adults. Arch Microbiol 2014;196:73-77.

54. Aguirre de Cárcer D, Cuív PO, Wang T, et al. Numerical ecology validates a biogeographical distribution and gender-based effect on mucosaassociated bacteria along the human colon. ISME J 2011;5:801-809.

55. Mueller S, Saunier K, Hanisch C, et al. Differences in fecal microbiota in different European study populations in relation to age, gender, and country: a cross-sectional study. Appl Environ Microbiol 2006;72:10271033.

56. Sheng L, Jena PK, Liu HX, et al. Gender differences in bile acids and microbiota in relationship with gender dissimilarity in steatosis induced by diet and FXR inactivation. Sci Rep 2017;7:1748.

57. Mu Q, Zhang $\mathrm{H}$, Liao X, et al. Control of lupus nephritis by changes of gut microbiota. Microbiome 2017;5:73.
58. Lee JY, Kim N, Nam RH, et al. Probiotics reduce repeated water avoidance stress-induced colonic microinflammation in wistar rats in a sexspecific manner. PLoS One 2017;12:e0188992.

59. Zhang H, Wang Z, Li Y, et al. Sex-based differences in gut microbiota composition in response to tuna oil and algae oil supplementation in a d-galactose-induced aging mouse model. Front Aging Neurosci 2018;10:187.

60. Karunasena E, McMahon KW, Chang D, Brashears MM. Host responses to the pathogen Mycobacterium avium subsp. paratuberculosis and beneficial microbes exhibit host sex specificity. Appl Environ Microbiol 2014;80:4481-4490.

61. Mahnic A, Rupnik M. Different host factors are associated with patterns in bacterial and fungal gut microbiota in Slovenian healthy cohort. PLoS One 2018;13:e209209.

62. Strati F, Di Paola M, Stefanini I, et al. Age and gender affect the composition of fungal population of the human gastrointestinal tract. Front Microbiol 2016;7:1227.

63. Edogawa S, Peters SA, Jenkins GD, et al. Sex differences in NSAIDinduced perturbation of human intestinal barrier function and microbiota. FASEB J 2018;32:6615-6625.

64. Son HJ, Kim N, Song CH, et al. Sex-related alterations of gut microbiota in the $\mathrm{C} 57 \mathrm{BL} / 6$ mouse model of inflammatory bowel disease. J Cancer Prev 2019;24:173-182.

65. Ober C, Loisel DA, Gilad Y. Sex-specific genetic architecture of human disease. 2008;9:911-922.

66. Claesson MJ, Cusack S, O'Sullivan O, et al. Composition, variability, and temporal stability of the intestinal microbiota of the elderly. Proc Natl Acad Sci USA 2011;108(suppl 1):4586-4591.

67. Rincel M, Aubert P, Chevalier J, et al. Multi-hit early life adversity affects gut microbiota, brain and behavior in a sex-dependent manner. Brain Behav Immun 2019;80:179-192.

68. Clarke G, Grenham S, Scully P, et al. The microbiome-gut-brain axis during early life regulates the hippocampal serotonergic system in a sexdependent manner. 2013;18:666-673.

69. Sisk CL, Foster DL. The neural basis of puberty and adolescence. Nat Neurosci 2004;7:1040-1047.

70. Biagi E, Nylund L, Candela M, et al. Through ageing, and beyond: gut microbiota and inflammatory status in seniors and centenarians. PLoS One 2010;5:e10667.

71. Lee SM, Kim N, Yoon H, Nam RH, Lee DH. Microbial changes and host response in F344 rat colon depending on sex and age following a high-fat diet. Front Microbiol 2018;9:2236.

72. Bischoff SC. Microbiota and aging. Curr Opin Clin Nutr Metab Care 2016;19:26-30.

73. Salles N. Basic mechanisms of the aging gastrointestinal tract. Dig Dis 2007;25:112-117.

74. Enck P, Zimmermann K, Rusch K, Schwiertz A, Klosterhalfen S, Frick JS. The effects of ageing on the colonic bacterial microflora in adults. $\mathrm{Z}$ Gastroenterol 2009;47:653-658.

75. De Filippo C, Cavalieri D, Di Paola M, et al. Impact of diet in shaping gut microbiota revealed by a comparative study in children from Europe and rural Africa. Proc Natl Acad Sci USA 2010;107:14691-14696. 
76. Lee HS, Cho YH, Park J, Shin HR, Sung MK. Dietary intake of phytonutrients in relation to fruit and vegetable consumption in Korea. J Acad Nutr Diet 2013;113:1194-1199.

77. Regu GM, Kim H, Kim YJ, et al. Association between dietary carotenoid intake and bone mineral density in Korean adults aged 30-75 years using data from the fourth and fifth korean national health and nutrition examination surveys (2008-2011). Nutrients 2017;9:1025.

78. Barrasa JI, Olmo N, Lizarbe MA, Turnay J. Bile acids in the colon, from healthy to cytotoxic molecules. Toxicol In Vitro 2013;27:964-977.

79. Rowland RHIR. Metabolic activities of the gut microflora in relation to cancer. Microb Ecol Health Dis 2000;12:179-185.

80. Louis P, Hold GL, Flint HJ. The gut microbiota, bacterial metabolites and colorectal cancer. Nat Rev Microbiol 2014;12:661-672.

81. Bolnick DI, Snowberg LK, Hirsch PE, et al. Individual diet has sex-dependent effects on vertebrate gut microbiota. Nat Commun 2014;5:4500.

82. Lee JR, Muckerman JE, Wright AM, et al. Sex determines effect of physical activity on diet preference: association of striatal opioids and gut microbiota composition. Behav Brain Res 2017;334:16-25.

83. Lee SM, Kim N, Yoon H, et al. Compositional and functional changes in the gut microbiota in irritable bowel syndrome patients. Gut Liver Published Online First: 28 March 2020. doi: 10.5009/gnl19379.

84. Kim YS, Kim N. Sex-gender differences in irritable bowel syndrome. J Neurogastroenterol Motil 2018;24:544-558.

85. Ait-Belgnaoui A, Payard I, Rolland C, et al. Bifidobacterium longum and Lactobacillus helveticus synergistically suppress stress-related visceral hypersensitivity through hypothalamic-pituitary-adrenal axis modulation.
J Neurogastroenterol Motil 2018;24:138-146.

86. Distrutti E, Monaldi L, Ricci P, Fiorucci S. Gut microbiota role in irritable bowel syndrome: new therapeutic strategies. World J Gastroenterol 2016;22:2219-2241.

87. Tremaroli V, Bäckhed F. Functional interactions between the gut microbiota and host metabolism. Nature 2012;489:242-249.

88. Rivière A, Selak M, Lantin D, Leroy F, De Vuyst L. Bifidobacteria and butyrate-producing colon bacteria: importance and strategies for their stimulation in the human gut. Front Microbiol 2016;7:979.

89. Encarnação JC, Abrantes AM, Pires A, Botelho MF. Revisit dietary fiber on colorectal cancer: butyrate and its role on prevention and treatment. Cancer Metastasis Rev 2015;34:465-478.

90. Vemuri R, Sylvia KE, Klein SL, et al. The microgenderome revealed: sex differences in bidirectional interactions between the microbiota, hormones, immunity and disease susceptibility. Semin Immunopathol 2019;41:265-275.

91. Ou J, Carbonero F, Zoetendal EG, et al. Diet, microbiota, and microbial metabolites in colon cancer risk in rural Africans and African Americans. Am J Clin Nutr 2013;98:111-120.

92. Lee JY, Kim N, Choi YJ, et al. Expression of tight junction proteins according to functional dyspepsia subtype and sex. J Neurogastroenterol Motil 2020;26:248-258.

93. Ferrario C, Taverniti V, Milani C, et al. Modulation of fecal clostridiales bacteria and butyrate by probiotic intervention with Lactobacillus paracasei DG varies among healthy adults. J Nutr 2014;144:1787-1796. 